\title{
WEBQUEST Y EDUBLOG: EXPERIENCIA EN LA ENSEÑANZA UNIVERSITARIA DE TOXICOLOGÍA DE ALIMENTOS
}

\author{
(WEBQUEST AND EDUBLOG: AN EXPERIENCE IN THE UNIVERSITY EDUCATION OF FOOD \\ TOXICOLOGY)
}

\author{
María Claudia Degrossi \\ Universidad de Belgrano (UB) \\ Susana Carnevali de Falke \\ Instituto Universitario de Ciencias de la Salud, (I.U.C.S) Fundación H.A. Barceló. \\ Buenos Aires (Argentina)
}

\section{RESUMEN}

El presente trabajo relata una experiencia educativa, con desarrollo de una webquest y creación de un edublog, realizada en el año 2007 durante el dictado de Toxicología de Alimentos de la Carrera en Nutrición en dos instituciones educativas. Se eligió como tema el Análisis de Riesgos, aplicado a edulcorantes no calóricos, por su carácter central en la asignatura y la gran cantidad de información disponible en la red sobre los mismos.

Se organizaron actividades grupales, dentro y fuera del aula, en tiempos flexibles, respetando los intereses de los alumnos, quienes debieron realizar presentaciones parciales y un trabajo final que reuniera los resultados y conclusiones de la investigación realizada.

La webquest resultó una herramienta organizadora de la actividad docente y de las tareas de los alumnos. Respecto al blog, favoreció el intercambio de información entre los grupos y motivó a los estudiantes, superándose rápidamente las dificultades iniciales en su utilización.

Palabras clave: webquest, edublog, análisis de riesgos, toxicología de alimentos.

\section{ABSTRACT}

The present work relates our 2007 educative experience with the development of a webquest and creation of an edublog in connection with a university course, "Food Toxicology of the Nutrition Carrier," 
conducted in two universities. The topic of "risk analysis applied to non caloric sweeteners," was chosen because of its central importance to the subject and the amount of information available in the network. Group activities were organized inside and outside the classroom, at flexible times according to the interests of the students who were required to make presentations and produce a final work that presented their research findings and conclusions. The webquest was deployed as an organizing tool for teaching activity and for the students' assignments. The blog facilitated exchange of information between groups and motivated students to overcome quickly the initial difficulties of its application.

Keywords: webquest, edublog, risk analysis, food toxicology.

En la sociedad de la información y del conocimiento, la enseñanza universitaria nos impone a los docentes un cambio de paradigma en nuestras prácticas educativas. Debemos buscar alternativas, mediante la incorporación y uso de las Nuevas Tecnologías, que nos permitan poner mayor énfasis en actividades dirigidas a “enseñar a pensar" o "aprender a aprender" (Area y col., 2008; Casado Ortiz, 2006; García-Valcárcel, 2007). La universidad, si bien busca adaptarse permanentemente a la evolución de los conocimientos científicos (Malendro y col., 2008), ya no puede proporcionar toda la información relevante. Como señala Fernández Aedo, "ésta es mucho más móvil y flexible que la propia institución” (Fernández Aedo y col., 2008). Además, en la era de las Nuevas Tecnologías de la Información y Comunicación (NTIC) que sin dudas han alcanzado a todas las esferas de la sociedad, desde la enseñanza a la práctica profesional y desde el mundo del arte a la investigación (Cabero, 2003), una enorme cantidad de información (y no sólo la útil y relevante) está disponible en la red.

\section{CONTEXTO DE LA ENSEÑANZA DE TOXICOLOGÍA DE ALIMENTOS}

Esta asignatura se caracteriza por su enorme extensión de contenidos, resultando imposible la existencia de un único libro que llegue a cubrirlos en su totalidad. Se destaca, además, la constante emergencia de nuevos contaminantes asociados a los alimentos y la rápida "globalización" de los problemas que ellos generan. La red se convierte entonces en una fuente constante de consulta para docentes, alumnos y profesionales en esta área de conocimiento. Sin embargo, puede comprobarse que en la Web circula información contradictoria, hecho que en ocasiones genera mucha confusión en los estudiantes.

Entonces, como docentes, debemos enseñar a utilizar las NTIC adecuadamente: acceder a la información, seleccionarla y validarla, proporcionándoles a nuestros 
alumnos, al mismo tiempo, las capacidades de aprendizaje que les permitan una asimilación crítica de la misma (Fernández Aedo y col., 2008).

\section{PROPUESTA PARA EL DICTADO DE LA ASIGNATURA EN AMBAS INSTITUCIONES}

Valorando el rol del nutricionista como comunicador de la salud, juzgamos oportuno dictar, en cada una de las instituciones en las que nos desempeñamos, los contenidos de la asignatura desde el enfoque innovador, el del Análisis de Riesgos. Esta metodología, permite realizar una evaluación del riesgo de una población ante el consumo de sustancias químicas a través de los alimentos, plantear posibles estrategias para su gestión y comunicar a la comunidad el riesgo encontrado y cómo reducirlo. Trabajar con este enfoque requiere que el alumno maneje una gran cantidad de términos y semitérminos precisos y propios de la disciplina.

Consideramos también, dado que se trata de enseñanza en el nivel superior, la necesidad de proponer a los estudiantes actividades de investigación, no sólo por tratarse de una potente herramienta de formación científica, sino como medio para promover el desarrollo de una mentalidad creadora, estimulando el análisis crítico de la realidad y la capacidad de síntesis y entusiasmándolos en la justificación de su verdad científica.

Nos planteamos especialmente, que a través del desarrollo de esta experiencia, los alumnos:

- Aprendieran los conceptos del Análisis de Riesgos (evaluación, gestión y comunicación de riesgos); utilizaran correctamente términos y semitérminos de la asignatura; e integraran los contenidos de la misma;

- Mejoraran su utilización de la Web 2.0;

- Utilizaran críticamente información de la red;

- Analizaran críticamente la información recabada y elaboraran un trabajo científico con la misma;

- Trabajasen colaborativamente;

- Aumentaran su comunicación, dentro y fuera del aula presencial;

- Reflexionaran acerca del proceso de aprendizaje y,

- Comprendieran el impacto de las NTIC en el mundo profesional. 
Todo lo expuesto nos llevó a reflexionar en el empleo de estrategias que permitieran la utilización de las nuevas tecnologías de manera intensa y provechosa y, paralelamente, facilitaran el aprendizaje de los temas centrales de la asignatura, desarrollando en los alumnos las capacidades de evaluador, gestor y comunicador de riesgo.

\section{FUNDAMENTOS DE LA ELECCIÓN DE EDUBLOG Y WEBQUEST COMO HERRAMIENTAS DIDÁCTICAS}

A través de algunos encuentros y de numerosas comunicaciones por correo electrónico, realizamos un análisis de las estrategias disponibles en la "era informática" e intercambiamos opiniones sobre las distintas alternativas posibles. Optamos entonces por la articulación de la enseñanza presencial con modalidades asincrónicas de comunicación a través de la creación de un edublog y, compatible con éste, una webquest.

\section{El edublog}

Dentro de las posibilidades que brinda la Web 2.0, encontramos que los edublogs, weblogs utilizados para apoyar el proceso de enseñanza y aprendizaje, por su accesibilidad e interactividad entre usuarios, eran un instrumento adecuado para el propósito de nuestro trabajo. Estos sitios Web, ofrecen diferentes recursos como: información básica sobre su contenido, finalidad y autor/autores del mismo, tareas a realizar por los alumnos, hiperenlaces a sitios Web con información específica sobre temas, entre otros, pudiendo muy bien adaptarse para coordinar tareas de investigación on-line, al actuar como eje vertebrador, ordenando y organizando las actividades. Representan además un modo de acceder, analizar, compartir y discutir la información, recibir retroalimentación y exponer dudas. De esta manera, generan un interesante juego de construcción conceptual donde, en muchos casos, se resignifica el conocimiento, se da lugar al debate y a la expansión de fuentes de información para la fundamentación de diferentes posturas respecto de los tópicos tratados en cada caso. Promueven el aprendizaje continuo, extendiendo el espacio áulico fuera de sus límites físicos y temporales (Bartolomé, 2008; Cabero, 2007; Lara, 2007; Peña y col., 2006; Salinas y Viticcioli, 2008).

Teniendo en cuenta estas características, consideramos apropiada la opción de un edublog en el que los estudiantes pudieran desarrollar a lo largo de un semestre un trabajo grupal, en etapas, y, de este modo, aprender colaborativamente sobre 
un tópico central de Toxicología de Alimentos. De esta manera lograríamos no sólo organizar los contenidos y recursos formativos, sino también mayor interacción entre los alumnos fuera del aula presencial. Consideramos que estos dos aspectos contribuirían a mejorar la calidad del proceso de enseñanza y de aprendizaje (Bartolomé, 2008; Cabero, 2007; Garcia Aretio, 2008).

\section{La webquest}

Encontramos a esta herramienta de gran utilidad, ya que constituye una nueva perspectiva referente al trabajo de los estudiantes dentro y fuera del aula. Es una actividad de investigación guiada con recursos principalmente de Internet, donde se establecen previamente todos los pasos a seguir, incluidos la distribución temporal y los recursos. Esta herramienta, siempre propone una tarea auténtica de modo que implica a los alumnos en la creación de un producto que repercute en el mundo real fuera del aula; fomenta la participación y favorece la utilización de habilidades cognitivas de alto nivel y, prioriza la transformación de la información permitiendo al alumno que elabore su propio conocimiento; además, se trata de un trabajo cooperativo en el que cada persona es responsable de una parte. Consta básicamente de las siguientes partes: Introducción; Tarea; Proceso; Recursos; Evaluación y Conclusiones, que resumen la experiencia y estimulan la reflexión acerca del proceso de tal manera que extienden y generalizan lo aprendido. (Rodera Bermúdez, 2008; Palacio Picos, 2009)

Acordamos entonces que una webquest sobre Análisis de Riesgos permitiría a los alumnos transitar los conceptos de la evaluación, la gestión y la comunicación de riesgos a través de tareas específicas. Además, al desarrollarla a largo plazo, daría el tiempo necesario a los estudiantes para apropiarse de la compleja terminología de este enfoque.

\section{Desarrollo de la Experiencia}

Se llevó a cabo en distintas etapas:

\section{Planificación y diseño de la webquest}

Para su desarrollo, y trabajando colaborativamente a través del correo electrónico, tuvimos en cuenta que la propuesta resultara: coherente, en cuanto a las posibilidades de interacción y de facilitación del aprendizaje; sistemática, 
organizando, estructurando, encadenando y relacionando todos sus componentes; rica, de manera que el conocimiento pudiese ser abordado desde fuentes y puntos de vista diferentes y relacionado a otros contenidos; real y atractiva, a fin de aumentar la motivación y acercar a los estudiantes a actividades relacionadas a su futuro profesional; y relevante, en cuanto al tratamiento de temas fundamentales e interesantes para su formación y experiencia personal (Palacio Picos, 2009). Por ello, elegimos como tema, ligado tanto al mundo laboral como cotidiano, el riesgo de la población frente al consumo de edulcorantes no calóricos. De esta manera quedaría planteado el problema a resolver ¿cuán seguros son los "límites seguros"?.

El diseño de la webquest quedó conformado de la siguiente manera:

- Introducción: presentación de la intención de integrar los conceptos teóricos de la asignatura mediante una actividad práctica en la cual realizarían los procesos de evaluación, gestión y comunicación de riesgos de edulcorantes sintéticos.

- Tarea: una investigación guiada, en etapas, a través de la cual cada grupo de estudiantes realizaría una evaluación del consumo deun edulcorante en particular, para luego, en función del riesgo detectado, proponer posibles estrategias de gestión y diseñar finalmente estrategias de comunicación del riesgo detectado a la ciudadanía.

- Proceso: presentación de la modalidad de trabajo, incluyendo un cronograma flexible, que incluía la forma de presentación, las posibles fechas y el tipo de actividad, para cada una de las cuales se prepararon consignas específicas.

- Recursos: una lista de páginas electrónicas y referencias bibliográficas recomendadas, que debía ser ampliada por los estudiantes.

- Evaluación: los criterios evaluativos, elaborados por cada cátedra, de modo de mostrar el camino a seguir, al conocer el estudiante, desde el comienzo, que se esperaba de él y como se evaluaría su trabajo. A continuación, se presentan los criterios: 


\begin{tabular}{|c|c|}
\hline Criterios & Descripción \\
\hline 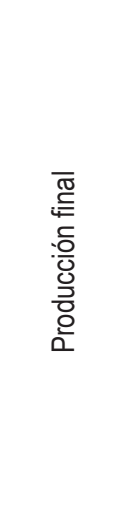 & $\begin{array}{l}\text { Excelente: Cumple las consignas en cuanto a los aspectos formales. La información está muy } \\
\text { bien organizada, es pertinente y relevante. Se observa un hilo conductor que guía a través del } \\
\text { texto, haciéndolo atractivo y ameno. Utiliza términos y semitérminos de la asignatura y los aplica } \\
\text { correctamente. Sustenta y fundamenta opiniones. Los diagramas y tablas son pertinentes. } \\
\text { Bueno: Cumple con parte de las consignas en cuanto a los aspectos formales. Existe } \\
\text { organización de la información, pero algunos párrafos no están bien redactados. Utiliza términos } \\
\text { y semitérminos de la asignatura y los aplica correctamente. Sustenta y fundamenta opiniones. } \\
\text { Existe un hilo conductor. Los diagramas y tablas son pertinentes. } \\
\text { Requiere Mejoras: No cumple con las consignas en cuanto a los aspectos formales. La } \\
\text { información no está bien organizada, la redacción no es clara, con oraciones poco relacionadas. } \\
\text { Utiliza escasamente términos y semitérminos de la asignatura o no los aplica correctamente. No } \\
\text { sustenta o fundamente opiniones. Utiliza escasamente diagramas o tablas. }\end{array}$ \\
\hline 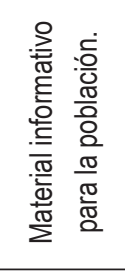 & $\begin{array}{l}\text { Excelente: Es excepcionalmente atractivo. La información está bien organizada, es correcta y } \\
\text { pertinente al grupo poblacional al cual está dedicada. } \\
\text { Bueno: Tiene un formato atractivo. La información está bien organizada, es correcta, pero no es } \\
\text { pertinente al grupo poblacional al cual está dedicada. } \\
\text { Requiere Mejoras: El formato del folleto y la organización del material es poco atractivo para el } \\
\text { lector. La información no es completa y no contempla la población hacia la cual va dirigida. }\end{array}$ \\
\hline 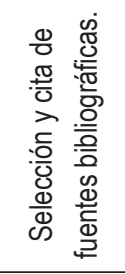 & $\begin{array}{l}\text { Excelente: Utiliza artículos científicos de los cuales extrae información para sustentar sus } \\
\text { opiniones. Todas las fuentes de información están documentadas en el formato correcto. } \\
\text { Bueno: Utiliza pocos artículos científicos. Todas las fuentes de información están documentadas, } \\
\text { pero muchas no están en el formato correcto. } \\
\text { Requiere Mejoras: No recurre a artículos científicos. Algunas fuentes de información no están } \\
\text { documentadas. }\end{array}$ \\
\hline 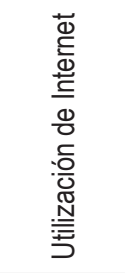 & $\begin{array}{l}\text { Excelente: Usa enlaces de Internet para encontrar información y selecciona sitios confiables. } \\
\text { Llega a analizar fuente, autor, calidad de imágenes, texto y rigor científico. } \\
\text { Bueno: Usa enlaces de Internet para encontrar información, pero recurre a algunos sitios no } \\
\text { confiables. Sólo analiza fuente y autor. } \\
\text { Requiere Mejoras: Usa enlaces de Internet para encontrar información, pero no es selectivo } \\
\text { respecto a los sitios. }\end{array}$ \\
\hline 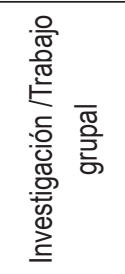 & $\begin{array}{l}\text { Excelente: El grupo está organizado, distribuye tareas y la integra en un material coherente. } \\
\text { Todos los integrantes pueden responder a todos los temas investigados. } \\
\text { Bueno: El grupo está organizado, distribuyen las tareas, pero la integración es pobre. Todos los } \\
\text { integrantes pueden responder a algunos de los temas investigados. } \\
\text { Requiere Mejoras: El grupo no tiene un plan claro para organizar la información y sólo algunos } \\
\text { de sus integrantes pueden responder a los temas investigados. }\end{array}$ \\
\hline
\end{tabular}

Tabla 1. Criterios de evaluación de la webquest en el IUCS 


\begin{tabular}{|c|c|}
\hline Categoría & Descripción \\
\hline$\frac{\stackrel{9}{N}}{\frac{d}{\infty}}$ & $\begin{array}{l}\text { Excelente: El trabajo final demuestra que los estudiantes se esforzaron al máximo. } \\
\text { Bueno: El trabajo final demuestra que los estudiantes no alcanzaron a poner todo su esfuerzo. } \\
\text { Requiere Mejoras: El trabajo final demuestra que los estudiantes no pusieron ningún } \\
\text { esfuerzo. }\end{array}$ \\
\hline$\frac{\frac{\pi}{0}}{\frac{0}{0}}$ & $\begin{array}{l}\text { Excelente: Se analizaron correctamente los conceptos y estuvieron bien aplicados. } \\
\text { Bueno: Se analizaron correctamente los conceptos, pero sólo se aplican en forma correcta en } \\
\text { forma parcial. } \\
\text { Requiere Mejoras: El análisis de los conceptos fue confuso, no se aplican correctamente. }\end{array}$ \\
\hline 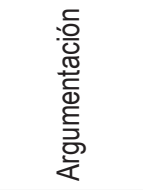 & $\begin{array}{l}\text { Excelente: La presentación impacta y es convincente. } \\
\text { Bueno: La presentación es convincente. } \\
\text { Requiere Mejoras: La presentación no es convincente. }\end{array}$ \\
\hline 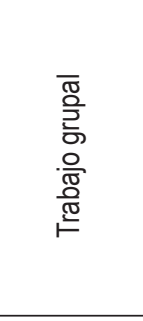 & $\begin{array}{l}\text { Excelente: Todos los estudiantes en el grupo pueden contestar adecuadamente todas las } \\
\text { preguntas relacionadas con la información en la presentación y el proceso técnico usado para } \\
\text { crearlo. } \\
\text { Bueno: Todos los estudiantes en el grupo pueden contestar adecuadamente la mayoría de las } \\
\text { preguntas relacionadas con la presentación y el proceso técnico usado para crearlo. } \\
\text { Requiere Mejoras: Varios estudiantes en el grupo parecen tener poco conocimiento sobre la } \\
\text { información y procesos técnicos usados en la presentación. }\end{array}$ \\
\hline 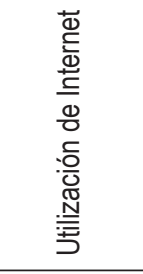 & $\begin{array}{l}\text { Excelente: Usa enlaces de Internet para encontrar información y selecciona sitios confiables. } \\
\text { Llega a analizar fuente, autor, calidad de imágenes, texto y rigor científico. } \\
\text { Bueno: Usa enlaces de Internet para encontrar información, pero recurre a algunos sitios no } \\
\text { confiables. Sólo analiza fuente y autor. } \\
\text { Requiere Mejoras: Usa enlaces de Internet para encontrar información, pero no es selectivo } \\
\text { respecto a los sitios. }\end{array}$ \\
\hline 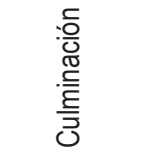 & $\begin{array}{l}\text { Excelente: Se completó el informe con todas las actividades en forma adecuada. } \\
\text { Bueno: Se completó el informe pero quedaron actividades deficientes o incompletas. } \\
\text { Requiere Mejoras: El informe quedó incompleto. Faltaron más de } 2 \text { actividades. }\end{array}$ \\
\hline
\end{tabular}

Tabla 2. Criterios de evaluación de la webquest en la UB

- Conclusión: reflexiones finales sobre lo realizado y las metas alcanzadas por los alumnos, luego de la discusión oral y publicación de los trabajos finales en el edublog. 


\section{Publicación de la webquest}

Para favorecer el intercambio entre grupos e introducir a los alumnos en el uso de una herramienta útil en sus vidas profesionales, publicamos la webquest en nuestros respectivos edublogs, cuyo diseño se muestra a continuación:

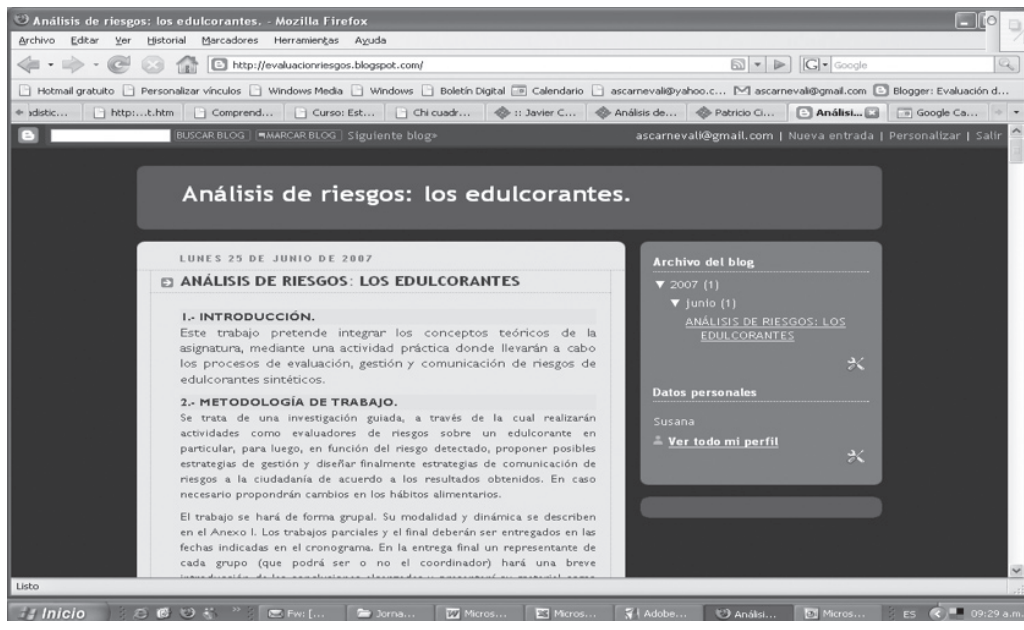

Figura 1. Edublog de la Cátedra de Microbiología y Parasitología Gral. y de Alimentos de la IUCS - Fundación Barceló

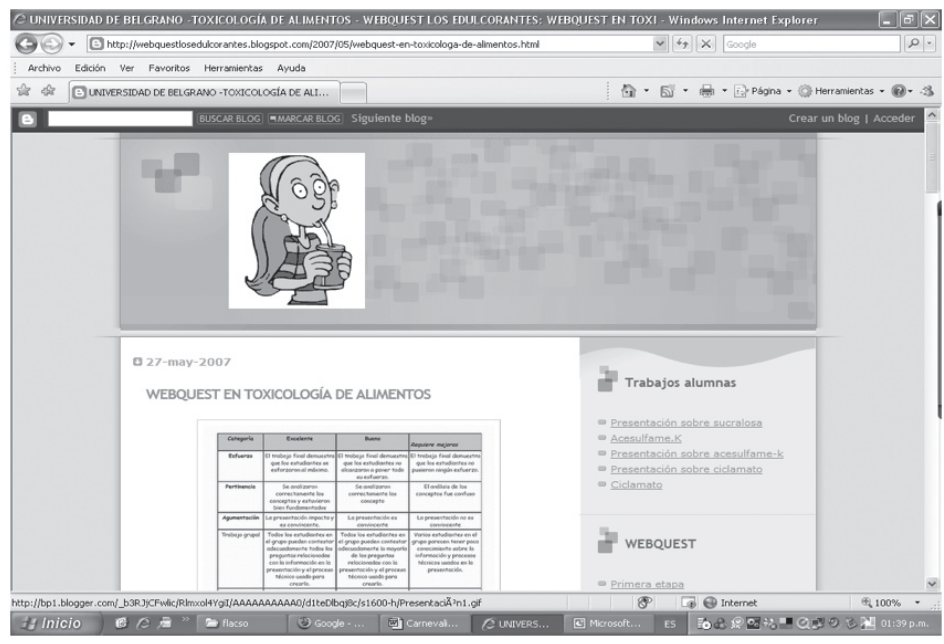

Figura 2. Edublog de la Cátedra de Toxicología de Alimentos - UB 


\section{Implementación}

La propuesta se desarrolló, en la Universidad de Belgrano, en el primer cuatrimestre con la intervención de 13 alumnos que conformaron 5 grupos de trabajo. En el IUCS, tuvo lugar en el segundo cuatrimestre, participando 27 alumnos del turno mañana y 19 del turno noche (12 grupos en total). Una parte de cada encuentro áulico, paralelo al desarrollo de la asignatura, se dedicó a trabajar sobre la investigación, con presentaciones orales de las producciones de los grupos correspondientes a cada etapa. Éstas también se publicaron en el blog de modo de fomentar el intercambio de información y la retroalimentación entre los distintos grupos y del docente. En la entrega final, un representante de cada grupo introdujo brevemente en las conclusiones alcanzadas y presentó el material como comunicador de riesgos.

\section{Evaluación de la experiencia}

Para valorar el alcance de los objetivos propuestos, utilizamos los criterios incluidos en la webquest y la autoevaluación de los alumnos.

En el IUCS, los estudiantes recibieron el último día de clases, una planilla de autoevaluación, de realización anónima y voluntaria. Contempló entre otros ítems, la opinión delos estudiantes sobrela metodología empleada, las dificultades encontradas y sugerencias para la mejora. Al tratarse de un grupo más reducido de alumnos en la Universidad de Belgrano, la evaluación sobre la experiencia fue realizada en un diálogo abierto con los estudiantes, tomándose nota de los comentarios.

Resultados obtenidos de la webquest

En la Universidad de Belgrano, 3/5 grupos alcanzaron resultados excelentes en los distintos aspectos evaluados; 1/5 alcanzó la calificación de Bueno y el otro grupo no cumplió con consignas del trabajo final y del material de comunicación al consumidor, debiendo rehacerlos.

Respecto al IUCS, los resultados se presentan a continuación: 


\begin{tabular}{|l|c|c|c|}
\hline Criterios & Excelente $^{1}$ & Bueno $^{1}$ & Requiere mejoras $^{1}$ \\
\hline Producción final & $9 / 12$ & $3 / 12$ & --- \\
\hline Material informativo para la población. & $7 / 12$ & $2 / 23$ & $3 / 12^{2}$ \\
\hline Selección y cita de fuentes bibliográficas. & $4 / 12$ & $8 / 12$ & --- \\
\hline Utilización de Internet & $9 / 12$ & $3 / 12$ & --- \\
\hline Investigación /Trabajo grupal & $12 / 12$ & --- & --- \\
\hline
\end{tabular}

${ }^{1}$ ( $N^{0}$ de grupos que alcanzó la calificación/ número total de grupos de trabajo)

${ }^{2}$ Estos 3 grupos no elaboraron el material.

Tabla 3. Resultados obtenidos en los 12 grupos de trabajo en el IUCS

De la lectura de los resultados, puede apreciarse que la mayoría de los grupos elaboró un informe final cumpliendo con las consignas establecidas en cuanto a los aspectos formales y organizando la información de manera pertinente y relevante. Se observó en éstos, en general, un hilo conductor que guiaba a través del texto, haciéndolo atractivo y ameno al lector.

Los términos y semitérminos de la asignatura se utilizaron y aplicaron correctamente, las opiniones se sustentaron y fundamentaron y las tablas utilizadas resultaron claras y pertinentes, en la mayoría de los casos.

La elaboración del material de comunicación de riesgos, fue sin duda uno de los puntos fuertes, al poder los estudiantes, durante su preparación, no sólo desarrollar toda su creatividad, sino también resumir los principales conceptos aprendidos, elaborando así un mensaje claro hacia la comunidad de los resultados sobre el riesgo a los que habían arribado.

Se detectaron algunas dificultades, como en la selección y cita de fuentes bibliográficas, ya que en general los alumnos utilizan escasamente los artículos científicos y, si bien las fuentes de información están documentadas, la mayoría de ellas no se encuentra en el formato correcto. Por otro lado, algunos grupos del IUCS no elaboraron el material informativo, ya que no leyeron totalmente las consignas formuladas. Lo mismo ocurrió con uno de los grupos de la Universidad de Belgrano. La lectura e interpretación de consignas es una cuestión que debe trabajarse permanentemente con los alumnos, ya que se advierte el problema de falta de atención a las mismas no sólo en este tipo de experiencias, sino también en la resolución de exámenes parciales y finales. 
Opiniones de los estudiantes sobre la experiencia

Del cuestionario de autoevaluación administrado a los alumnos y la evaluación oral de la experiencia surgieron los siguientes comentarios:

"Trabajar en grupo, me resulta agradable porque me permite tener intercambio de opinión con compañeros".

"El trabajo en grupo es importante para poder ayudarnos entre todos y entender mejor la materia".

"Entre todos pudimos resolver las dificultades que se nos presentaron en el uso del blog".

"El poder interactuar con nuestros pares o con la profesora nos ayudó a comprender mejor"

"Realizar una investigación y relacionarla con la vida cotidiana nos facilitó el aprendizaje de los temas"

"De esta manera, el aprendizaje de los temas es más dinámico y entretenido"

"Aunque al principio resulta dificultosa la comprensión, una vez que se logra gracias a la interacción docente-alumno y alumno-alumno, los conocimientos son mas efectivos"

"Esta metodología nos permite una mejor visión de la realidad y comprensión de los problemas que causan los agentes estudiados"

"El trabajar de esta forma, genera mas dinamismo y permite profundizar los temas ampliando el conocimiento de forma mas práctica"

"Me pareció importante este enfoque ya que aplicar los conocimientos sirve para fijar los conceptos"

"Prefiero esta metodología, porque es con lo que nos vamos a encontrar diariamente cuando seamos profesionales, uno puede tener la teoría pero no saber aplicarla" 
"Esta metodología es interesante porque se crea mas compromiso y permite interactuar con los otros"

"Permite aplicar conocimientos". "Es más fácil aplicarlo a la realidad por los casos estudiados”.

Emergencia de estrategias metacognitivas

En la medida que se avanzó en la experiencia, se comprobó que los alumnos comenzaron a reflexionar sobre su propio aprendizaje. Dichas reflexiones fueron favorecidas por nuestra intervención docente frente al cuestionamiento, por parte de los alumnos, sobre cómo interpretar las consignas y por el problema planteado que la investigación debía resolver. Resultó también esencial el proceso de evaluación. El mismo implicó, en cada etapa, una primera presentación en forma oral, durante la cual facilitamos la discusión entre los distintos grupos y solicitamos la defensa de las distintas posiciones de los grupos frente a los resultados presentados. La técnica de la repregunta, utilizada para corregir cada informe, generó otra instancia de discusión oral y justificación de las respuestas, de los datos utilizados y el método seguido. Asimismo, la integración de la investigación en una presentación final favoreció la percepción de los alumnos de los logros alcanzados por cada uno de los grupos y el "para qué" de la asignatura, entendiendo su significado en su futuro quehacer laboral.

Algunos comentarios de los estudiantes que apoyan este resultado son:

"El trabajo nos sirvió más allá de aprender sobre el tema"

"Me sirvió para mejorar mi rendimiento en otras asignaturas"

"Poder completar este trabajo me dio seguridad en el estudio"

"Con la búsqueda de información se aprende muchísimo. Con la interpretación se aprenden a sacar muchas más conclusiones”.

"Mejoró notablemente mi capacidad de comprensión ya sea casos, textos, búsqueda a través de Internet, etc. Me resultó difícil pero logré sacar provecho a la materia". 
"Pienso que es la mejor manera de aprender y retener los conocimientos al ir resolviendo las dudas que surgen". "Me permitió integrar los conocimientos estudiados".

"Aprender las diferentes vías de búsqueda y la gran ramificación de los temas, me permitió encontrar la relación que existe con otras áreas". "Llegué a aplicarlo mucho a mi vida cotidiana, relaciono mucho la materia con todo lo que veo". "Relacionar la teoría con casos verídicos me sirvió para entender mejor y el material era accesible". "Es una metodología didáctica y que nos traslada más a la realidad a través del estudio de casos reales, donde uno se enriquece con las distintas opiniones que van surgiendo para resolver el caso. Permite desarrollar el pensamiento".

\section{CONCLUSIONES}

La integración de la webquest y el edublog resultó de enorme utilidad en la enseñanza y el aprendizaje de Toxicología de Alimentos en los estudiantes de la Licenciatura en Nutrición de ambas instituciones. Durante la experiencia, se vivieron situaciones significativas de las cuales trascendieron las dificultades y fortalezas en el uso de ambas herramientas, tanto para los estudiantes como para nosotras.

Sin dudas, la webquest, por sus características, resultó una herramienta organizadora de las tareas de los alumnos y favoreció el trabajo colaborativo, ordenado y productivo, dentro y fuera del aula presencial. Además, entusiasmó a los estudiantes para la realización de las tareas, pudiendo todos los integrantes de cada grupo responder sobre los temas investigados. Las dudas e inquietudes surgidas durante el desarrollo de la webquest los impulsaron a seguir trabajando ya que comprobaban, en las distintas etapas, que todos los grupos se enfrentaban a situaciones inciertas y desconocidas, por la naturaleza del problema a resolver.

Respecto al blog, también favoreció el trabajo colaborativo con una fuerte interacción y un intercambio productivo de información entre los grupos. El hecho de posibilitarles ver sus producciones en la Web y compartirlas con sus familias, contribuyó a la motivación de los estudiantes. Este espacio, además de los encuentros del aula presencial, les dio la oportunidad de presentar y reflexionar sobre posibles estrategias para dar solución al problema planteado.

La experiencia en su conjunto, claramente, favoreció el proceso de metacognición, ya que al mejorar sus habilidades de comprensión, los alumnos aprendieron a evaluar 
su propio rendimiento y desarrollaron una perspectiva interna sobre el proceso de aprendizaje, alcanzando una mayor autonomía.

En relación a nuestro quehacer docente, consideramos que la webquest contribuyó enormemente a su organización, resultándonos sencillo su diseño e implementación. Por su parte, el blog nos permitió analizar conjuntamente las producciones parciales y el trabajo final de los estudiantes, no sólo para llevar a cabo su evaluación y calificación, sino también hacer una reflexión crítica de la propuesta, de sus bondades y de sus puntos problemáticos, y así realizar los cambios pertinentes para las futuras experiencias. Por ejemplo, revisar la claridad de las consignas, ajustar el cronograma, entre otros puntos.

Aunque la creación del blog fue muy rápida y no nos presentó dificultades, el máximo aprovechamiento de esta herramienta nos requirió un tiempo mayor de dedicación. Detodos modos, cabe destacar que existen disponibles en la red numerosos tutoriales que facilitaron nuestro aprendizaje sobre su óptima utilización.

Si bien la propuesta incrementó significativamente nuestra labor docente, fue una fuente muy intensa de motivación también para nosotras. Además de enseñar Toxicología de Alimentos de un modo más atractivo y provechoso para los alumnos, nos permitió ayudarlos a desarrollar comprensión sobre su actividad como comunicadores de la salud; orientarlos en el uso de las nuevas tecnologías, contribuyendo a la formación de usuarios críticos; orientarlos en cómo procesar la enorme cantidad de información disponible en la red, a validarla, a dotarla de sentido, de modo que se convirtieran en verdaderos contenidos de aprendizaje; a mostrar el impacto de las nuevas tecnologías en el campo de la asignatura, y además, ayudarlos en la utilización de las distintas posibilidades de comunicación que éstas brindan.

Para finalizar, consideramos que la realización del trabajo en forma conjunta en ambas instituciones, enriqueció nuestra profesión docente, pudiendo discutir las dudas y compartir temores o alegrías, tanto en forma previa, como durante y luego de la experiencia. 


\section{REFERENCIAS BIBLIOGRÁFICAS}

Area, M. (2003). WebQuest. Una estrategia de aprendizaje por descubrimiento basada en el uso de Internet: Guia didáctica para docentes [en línea]. Disponible en: http://webpages.ull.es/ users/manarea/webquest/] [Consulta 2009, 30 de Enero]

Area, M; Sanabria, A. L.; Gónzalez, M. (2008). Análisis de una experiencia de docencia universitaria semipresencial desde la perspectiva del alumnado. RIED - Revista Iberoamericana de Educación a Distancia. Volumen $11 \mathrm{~N}^{\mathrm{O}} 1$ Junio. [en línea]. Disponible en: http://www.utpl. edu.ec/ried/images/pdfs/volumen11/ manuel-area.pdf [Consulta 2009, 7 de Febrero]

Bartolomé, A. (2008). Entornos de aprendizaje mixto en educación superior. RIED - Revista Iberoamericana de Educación a Distancia. Volumen $11 \mathrm{~N}^{\mathrm{o}} 1$ Junio. [en línea]. Disponible en: http:// www.utpl.edu.ec/ried/images/pdfs/ volumen11/bartolome.pdf [Consulta 2009, 7 de Febrero]

Cabero Almenara, J.; Castaño Garrido, C.; Cebreiro López, B.; Gisbert Cervera, M.; Martínez Sánchez, F.; Morales Lozano, J. A.; Prendes Espinosa, M. P.; Romero Tena, R.; Salinas Ibáñez, J. (2003). Las nuevas tecnologías en la educación universitaria [en línea]. Disponible en: http://tecnologiaedu.us.es/bibliovir/ pdf/nnttact.pdf [Consulta 2009, 30 de Enero]

Cabero Almenara, J.; Llorente Cejudo, M.C. (2007). La interacción en el aprendizaje en red: uso de herramientas, elementos de análisis y posibilidades educativas. RIED - Revista Iberoamericana de Educación a Distancia. Volumen $10 \mathrm{~N}^{\mathrm{o}}$ 2 Diciembre. [en línea]. Disponible en: http://www.utpl.edu.ec/ried/images/ pdfs/volumendiez/la-interaccion.pdf [Consulta 2009, 7 de Febrero]
Casado Ortiz, R. (2006). Convergencia con Europa y cambio en la universidad. Los profesores y las nuevas tecnologías como elementos clave en el nuevo modelo de aprendizaje del Espacio Europeo de Educación Superior. EDUTEC, Revista Electrónica de Tecnología Educativa. Núm. 20/ Enero [en línea]. Disponible en: http://www.uib.es/depart/gte/ gte/edutec-e/revelec20/casado20.htm [Consulta 2009, 7 de Febrero]

Fernández Aedo, R. R.; Carballos Ramos, E. (2008). Un modelo de autoaprendizaje con integración de las TIC y los métodos de gestión del conocimiento. Revista Iberoamericana de Educación a Distancia, vol. 11, $\mathrm{n}^{\mathrm{o}} 2$ Diciembre [en línea]. Disponible en: http:// www.utpl.edu.ec/ried/images/pdfs/ volumen11N2/modelodeautoaprendizje. pdf [Consulta 2009, 30 de Enero]

García Aretio, L. (2008). Rasgos de la EaD de siempre. Boletín Electrónico de Noticias de Educación a Distancia (BENED). Septiembre [en línea]. Disponible en: http://www.uned.es/catedraunescoead/editorial/p7-9-2008.pdf [Consulta 2009, 7 de Febrero]

García-Valcárcel, A. (2007). Herramientas tecnológicas para mejorar la docencia universitaria. Una reflexión desde la experiencia y la investigación. RIED - Revista Iberoamericana de Educación a Distancia. Volumen $10 \mathrm{~N}^{\mathrm{O}}$ 2 Diciembre [en línea]. Disponible en: http://www.utpl.edu.ec/ried/images/ $\mathrm{pdfs} /$ volumendiez/herramientastecnologicas.pdf [Consulta 2009, 7 de Febrero]

Lara, T. (2007). Los blogs como motor de la Universidad 2.0 en su décimo aniversario. [en línea]. Disponible en: http://turan.uc3m.es/uc3m/serv/GPC/ opinblogs.html [Consulta 2009, 7 de Febrero] 
Melendro, M.; Murga, M. A.; Novo, M.; Bautista-Cerro, M.J. (2008). Estrategias formativas innovadoras en educación ambiental y para el desarrollo sostenible. Revista Iberoamericana de Educación a Distancia, vol. 11, No 2 Diciembre [en línea]. Disponible en: http://www.utpl.edu. ec/ried/images/pdfs/volumen11N2/ estrategiasformativas.pdf [Consulta 2009, 30 de Enero]

Palacio Picos, A. (2009). Las webquest como estrategias metodológicas ante los retos de la convergencia europea de educación superior. Píxel Bit, Revistas de Medios y Comunicación, $\mathrm{N}^{\mathrm{o}} 34$ [en línea]. Disponible en: http://www.sav.us.es/ pixelbit/actual/16.pdf [Consulta 2009, 7 de Febrero]

Peña, I.; Córcoles, C. P.; Casado, C. (2006). El Profesor 2.0: docencia e investigación desde la Red. UOC Papers. Revista sobre la sociedad del conocimiento $\mathrm{N}^{\mathrm{O}}$ 3 [en línea]. Disponible en: http://www. uoc.edu/uocpapers/3/dt/esp/pena corcoles casado.pdf [Consulta 2009, 7 de Febrero]

Rodera Bermúdez,A. M.(2008). Catalogación y valoración de las webquests desde el área de educación física y el tratamiento de temáticas transversales. EDUTEC, Revista Electrónica de Tecnología Educativa. Núm. 27/ Noviembre [en línea]. Disponible en:

http://edutec.rediris.es/Revelec2/ revelec27/articulos_n27_PDF/EdutecE Rodera n27.pdf [Consulta 2009, $7 \mathrm{de}$ Febrero]

Salinas, M. I.; Viticcioli, S. M. (2008). Innovar con blogs en la enseñanza universitaria presencial.EDUTEC, RevistaElectrónica de Tecnología Educativa. Núm. 27/ Noviembre [en línea]. Disponible en: http://edutec.rediris.es/Revelec2/ revelec27/articulos n27 PDF/EdutecE MISanilas Viticcioli_n27.pdf [Consulta 2009, 7 de Febrero]

\section{PERFIL ACADÉMICO Y PROFESIONAL DE LAS AUTORAS}

María Claudia Degrossi. Dra. en Ciencias Químicas de la UBA. Realizó estudios de especialización en docencia en la FLACSO, en la Agencia Interamericana para la Cooperación y el Desarrollo (AICD/OEA) y en la Carrera Docente del Instituto Universitario de Ciencias de la Salud - Fundación Barceló. Participó en Foros nacionales e internacionales relacionados con Educación y Nuevas Tecnologías. Actualmente se desempeña como Profesora Asociada de Toxicología de Alimentos de la Universidad de Belgrano.

E-mail: cdebal@gmail.com

Susana Carnevali de Falke. Bioquímica (UBA). Diplomada Universitaria en Diseño y Gestión de Proyectos en e-learning y Educación a Distancia (Universidad de San Martín). Cursó estudios de especialización en FLACSO, en la Agencia Interamericana para la Cooperación y el Desarrollo (AICD/OEA) y en la Carrera Docente del IUCS - Fundación Barceló. Participó en diversos Foros nacionales e internacionales relacionados con Educación y Nuevas Tecnologías. Actualmente es 
Profesora Adjunta de Microbiología y Parasitología Gral. y de Alimentos del IUCS

- Fundación Barceló.

E-mail: ascarnevali@gmail.com

DIRECCIÓN DE LOS AUTORES

\author{
Zapata 535 \\ (CP 1426). Ciudad Autónoma de Buenos \\ Aires \\ Argentina \\ Padre Carranza 1475 \\ (CP 1682) \\ Villa Bosch - Pcia. Buenos Aires \\ Argentina
}

Fecha de recepción del artículo: 29/04/08

Fecha de aceptación del artículo: 09/02/09 